\title{
SOME EQUIVALENT NORMS ON THE HILBERT SPACE
}

\author{
G. ANDROULAKIS, F. SANACORY*
}

\begin{abstract}
We present a family of some new Tsirelson-type norms for the separable Hilbert space. Our results extend some results of S. Bellenot, J. Bernués and I. Deliyanni and provide candidates for distorted norms on the Hilbert space.
\end{abstract}

In this note we present a family of some new Tsirelson-type norms for the separable Hilbert space $\ell_{2}$. The motivation for presenting these norms is the following question of E. Odell and Th. Schlumprecht [1] which is also mentioned by T.W. Gowers [5]:

Question 1. Is it possible, for $\lambda>0$ to explicitly define an equivalent norm $|\cdot|$ on $\ell_{2}$ such that every infinite dimensional subspace $Y$ of $\ell_{2}$ contains two vectors $y_{1}$ and $y_{2}$ with $\left\|y_{1}\right\|_{2}=\left\|y_{2}\right\|_{2}=1$ (where $\|\cdot\|_{2}$ denotes the usual norm of $\ell_{2}$ ) and $\left|y_{1}\right| /\left|y_{2}\right|>\lambda$ ?

An implicitly defined norm with the above property exists by the solution of the famous distortion problem by Odell and Schlumprecht $[1,2]$. The family of norms that we present gives candidates for the solution of Question 1. Some of the norms of our family were first presented by S. Bellenot [3] which recently A.M. Pelczar [6] proved that these norms do not answer Question 1. Another purpose of present note, is to extend some results of Bellenot [3], J. Bernués and I. Deliyanni [4].

In order to define the new norms on $\ell_{2}$ we first introduce some notation. For $x=(x(i)) \in \ell_{2}$ and $E \subseteq \mathbb{N}$ we denote by $E x$ the natural projection of $x$ on $E$, i.e. $E x=((E x)(i))$ where $(E x)(i)=x(i)$ for all $i \in E$ and $(E x)(i)=0$ otherwise. Let $c_{00}$ be the vector space of scalar sequences with finite support. Set

$$
\mathcal{A}=\left\{y=(y(1), y(2), \ldots, y(N)) \in(0, \infty)^{N}: N \in \mathbb{N}, N \geq 2, \sum_{i=1}^{N}|y(i)|^{2}=1\right\} .
$$

We define a sequence of equivalent norms $\|\cdot\|_{y}$, for $y \in \mathcal{A}$, on $\ell_{2}$ as follows. Fix $y=$ $(y(i))_{i=1}^{N} \in \mathcal{A}$. Then $\|\cdot\|_{y}$ is the unique norm which satisfies

$$
\|x\|_{y}=\sup \sum_{i=1}^{N} y(i)\left\|E_{i} x\right\|_{y} \vee\|x\|_{\infty} \text { for every } x \in c_{00},
$$

where the supremum is taken with respect to any sequence of sets $E_{1}<E_{2}<\cdots<E_{N}$. Notice that in the definition of $\|\cdot\|_{y}$ we allow the sets $E_{i}$ to be empty and we adopt the convention that " $A<\emptyset$ " and " $\emptyset<B$ " are valid for any $A, B \subseteq \mathbb{N}$. It is standard to show that for every $y \in \mathcal{A}$ there exists a unique norm $\|\cdot\|_{y}$ which satisfies (1). We provide two equivalent definitions of the $\|\cdot\|_{y}$. Firstly, for every fixed $y \in \mathcal{A}$ we define a sequence of

Date: October 10, 2006.

1991 Mathematics Subject Classification. 46B03.

*The present paper is part of the Ph.D thesis of the second author which is prepared at the University of South Carolina under the supervision of the first author. 
norms $\|\cdot\|_{y}^{(n)}$ for $n \in \mathbb{N} \cup\{0\}$ which increases to the norm $\|\cdot\|_{y}$, as follows. Let $\|\cdot\|_{y}^{(0)}=\|\cdot\|_{\infty}$ (the $\ell_{\infty}$ norm). If for $n \in \mathbb{N} \cup\{0\}$ the $\|\cdot\|_{y}^{(n)}$ has been defined, then we define

$$
\|x\|_{y}^{(n+1)}=\sup \sum_{i=1}^{N} y(i)\left\|E_{i} x\right\|_{y}^{(n)} \vee\|x\|_{y}^{(n)} \text { for } x \in c_{00},
$$

where the supremum is taken with respect to any sequence of sets $E_{1}<E_{2}<\cdots<E_{N}$. Then $\|\cdot\|_{y}$ is the (pointwise) limit of $\|\cdot\|_{y}^{(n)}$ for $n \in \mathbb{N} \cup\{0\}$, and it satisfies (1).

Secondly, we define a sequence of subsets $K_{n}, n=0,1,2, \ldots$ of $c_{00}$ as follows. Let

$$
K_{0}=\left\{\lambda e_{i}: i \in \mathbb{N},|\lambda|=1\right\} .
$$

If $K_{n}$ has been defined for some $n \in \mathbb{N} \cup\{0\}$ then

$$
K_{n+1}=K_{n} \cup\left\{\sum_{i=1}^{N} y(i) x_{i}: x_{1}<x_{2}<\cdots<x_{N} \text { and } x_{i} \in K_{n} \text { for all } i \in\{1, \ldots, N\}\right\} .
$$

Let $K=\cup_{n=1}^{\infty} K_{n}$. Then, for every $x=(x(i)) \in c_{00}$ we have that

$$
\|x\|_{y}=\sup _{z=(z(i)) \in K} \sum_{i=1}^{\infty} x(i) z(i) .
$$

By using the Cauchy-Schwartz inequality it is easy to show by induction on $n \in N \cup\{0\}$ that $\|x\|_{y}^{(n)} \leq\|x\|_{2}$ for every $x \in c_{00}$, or $\|z\|_{2} \leq 1$ for all $z \in K_{n}$. Thus we have that $\|x\|_{y} \leq\|x\|_{2}$ for every $x \in c_{00}$. We will also show in Theorem 3 that $\|\cdot\|_{y}$ is in fact equivalent to $\|\cdot\|_{2}$.

For the proof of our main result we will need the notions of the $N$-tree, (where $N$ is an integer larger than 1), the tree decomposition of an interval and the tree decomposition of a function.

Definition 2. (a) Let $N$ be an integer larger than 1. An $N$-tree is a subset of $\{\emptyset\} \cup$ $\cup_{k=1}^{\infty}\{1,2, \ldots, N\}^{k}$ endowed with an order $\prec$, satisfying the following:

(i) $\emptyset \in \mathcal{T}$ and $\emptyset \prec t$ for all $t \in \mathcal{T} \backslash\{\emptyset\}$.

(ii) If $1 \leq k \leq \ell, n_{i} \in\{1, \ldots, N\}$ for $1 \leq i \leq \ell$ and $\left(n_{1}, \ldots, n_{\ell}\right) \in \mathcal{T}$ then $\left(n_{1}, \ldots, n_{k}\right) \in \mathcal{T}$

(iii) If $k, \ell \in \mathbb{N}$ and $n_{i}, m_{i} \in\{1, \ldots, N\}$ then $\left(n_{1}, \ldots, n_{k}\right) \prec\left(m_{1}, \ldots, m_{\ell}\right)$ if and only if $k<\ell$ and $m_{i}=n_{i}$ for $1 \leq i \leq k$.

A tree may be finite (respectively infinite) if it has finitely (respectively infinitely) many nodes. A node $t \in \mathcal{T}$ will be called maximal if there is no $s \in \mathcal{T}$ with $t \prec s$. If $t \in \mathcal{T} \backslash\{\emptyset\}$ we denote by $t^{-}$the unique immediate predecessor of $\mathcal{T}$. We denote by $\max (\mathcal{T})$ the set of maximal nodes of $\mathcal{T}$. If $t \in \mathcal{T} \backslash \max (\mathcal{T})$ then $t^{+}$will denote the set of the immediate successors of $t$, namely $t^{+}=\left\{s \in \mathcal{T}: t \prec s\right.$ and there is no $t^{\prime} \in$ $\mathcal{T}$ with $\left.t \prec t^{\prime} \prec s\right\}$.

(b) Let $\mathcal{T}$ be an $N$-tree for some $N \in \mathbb{N}, N>1$. By a tree decomposition or $\mathcal{T}$ decomposition of an interval $E \subset \mathbb{R}$ we mean a family of intervals $\left(E_{t}\right)_{t \in \mathcal{T}}$ indexed by the tree $\mathcal{T}$, satisfying the following:

(i) $E_{\emptyset}=E$.

(ii) Let $1 \leq n<m \leq N$. If $(n),(m) \in \mathcal{T}$ then $E_{(n)}<E_{(m)}$. If $k \in \mathbb{N}, n_{1}, \ldots, n_{k} \in$ $\{1, \ldots, N\}$ and $\left(n_{1}, \ldots, n_{k}, n\right),\left(n_{1}, \ldots, n_{k}, m\right) \in \mathcal{T}$ then $E_{(t, n)}<E_{(t, m)}$, where for $t=\left(n_{1}, \ldots, n_{k}\right) \in \mathcal{T}$ and $n \in\{1, \ldots, N\}$ we write $(t, n)$ to denote $\left(n_{1}, \ldots, n_{k}, n\right)$. 
(iii) $E_{t} \supseteq \cup\left\{E_{(t, i)}:(t, i) \in \mathcal{T}, i \in\{1, \ldots, N\}\right\}$.

(c) Let $\mathcal{T}$ be an $N$-tree for some $N \in \mathbb{N}, N>1$. By a tree decomposition or $\mathcal{T}$ decomposition of a function $g:[0, \infty) \rightarrow \mathbb{R}$ we mean a family of functions $\left(g_{t}\right)_{t \in \mathcal{T}}$ indexed by the tree $\mathcal{T}$, satisfying the following two properties.

(i) $g_{\emptyset}=g$.

(ii) If $t \in \mathcal{T} \backslash \max (\mathcal{T})$ and $t^{+}=\{(t, i): i \in F\}$ for some $F \subseteq\{1, \ldots N\}$ then $g_{t}=\sum_{i \in F} y(i) g_{(t, i)}$ with $\operatorname{supp}\left(g_{t, i}\right)<\operatorname{supp}\left(g_{t, j}\right)$ for every $i, j \in F$ with $i<j$.

If $g$ has a $\mathcal{T}$-decomposition $\left(g_{t}\right)_{t \in \mathcal{T}}$ and $I \subseteq \max (\mathcal{T})$ then there is a unique tree $\widetilde{\mathcal{T}} \subseteq \mathcal{T}$ such that $\max (\widetilde{\mathcal{T}})=I$. Let $S:=\cup_{t \in I}$ supp $g_{t}$ and $f:=g \uparrow_{S}$ be the restriction of $g$ on $S$. Then $f$ has a $\widetilde{\mathcal{T}}$-tree decomposition $\left(f_{t}\right)_{t \in \widetilde{\mathcal{T}}}$ which is naturally inherited by the $\mathcal{T}$-decomposition of $g$, if we set $f_{t}:=g_{t}$ for all $t \in I$ and $f_{t}:=g_{t} \uparrow_{s}$ for all $t \in \widetilde{\mathcal{T}} \backslash \max (\widetilde{\mathcal{T}})$.

Theorem 3. For every $y \in \mathcal{A},\|\cdot\|_{y}$ is equivalent to the norm of $\ell_{2}$.

Moreover, for $y=(y(i))_{i=1}^{N} \in \mathcal{A}$, let $a:=\min _{1 \leq i \leq N} y(i), b:=\max _{1 \leq i \leq N} y(i)$ and $M \in \mathbb{N}$ such that $b^{M} \leq a$. We have that $\|\cdot\|_{y} \leq\|\cdot\|_{2} \leq(M+4) N\|\cdot\|_{y}$.

Proof. Fix $y=(y(i))_{i=1}^{N} \in \mathcal{A}$. We consider the isometric embedding $\phi:\left(\ell_{2},\|\cdot\|_{2}\right) \rightarrow$ $\left(L_{2}[0, \infty),\|\cdot\|_{2}\right)$ defined by $\phi(x)=\sum_{i=1}^{\infty} x(i) \chi_{[i-1, i)}$ where $x=(x(i)) \in \ell_{2}$ and $\chi_{E}$ denotes the characteristic function of a set $E$. Here, by abuse of notation, $\|\cdot\|_{2}$ denotes the usual Hilbert space norm on both $\ell_{2}$ and $L_{2}[0, \infty)$. Let $\mathcal{B}$ denote the set of functions in $L_{2}[0, \infty)$ with bounded support, where for a function $f \in L_{2}[0, \infty)$ the support of $f$, supp $(f)$, is defined to be the essential support of $f$. Obviously we have that $\phi\left(c_{00}\right) \subset \mathcal{B}$. We divide the proof into three steps. In Step 1 we define a norm $|\cdot|_{y}$ on $\mathcal{B}$ such that the restriction $\phi \uparrow_{c_{00}}$ of $\phi$ on $c_{00}, \phi \Gamma_{c_{00}}:\left(c_{00},\|\cdot\|_{y}\right) \rightarrow\left(\mathcal{B},|\cdot|_{y}\right)$ is an isometric embedding. In Step 2 we define a norm \|\|$\cdot\|\|_{y}$ on $L_{2}[0, \infty)$ and we prove that it is equal to the usual norm $\|\cdot\|_{2}$ of $L_{2}[0, \infty)$. In Step 3 we prove that the norms $|\cdot|_{y}$ and $\||\cdot|\|_{y}$ are equivalent. Of course, these three steps finish the proof.

Step 1: Define a norm $|\cdot|_{y}$ on $L_{2}[0, \infty)$ in terms of its dual ball, as follows. Let

$$
L_{0}=\left\{f \in L_{2}[0, \infty):\|f\|_{2} \leq 1 \text { and } \operatorname{supp}(f) \subseteq[n-1, n) \text { for some } n \in \mathbb{N}\right\} .
$$

If $n \in \mathbb{N} \cup\{0\}$ and $L_{n}$ has been defined, then

$$
\begin{aligned}
L_{n+1}= & L_{n} \cup\left\{\sum_{i=1}^{N} y(i) f_{i}: f_{i} \in L_{n} \text { for all } i \text { and there exists }\left(n_{i}\right) \subseteq \mathbb{N}\right. \text { such that } \\
& \left.\operatorname{supp}\left(f_{1}\right)<n_{1} \leq \operatorname{supp}\left(f_{2}\right)<n_{2} \leq \operatorname{supp}\left(f_{3}\right)<\ldots<n_{N-1} \leq \operatorname{supp}\left(f_{N}\right)\right\} .
\end{aligned}
$$

Let $L=\cup_{n=0}^{\infty} L_{n}$ and for $f \in L_{2}[0, \infty)$ define

$$
|f|_{y}=\sup _{g \in L} \int_{0}^{\infty} f g .
$$

Notice that $L \subset \mathcal{B}$ and for every $g \in L$ we have that $\|g\|_{2} \leq 1$. Thus $|\cdot|_{y}$ is well defined and $|\cdot|_{y} \leq\|\cdot\|_{2}$. We now prove that $\phi \uparrow_{c_{00}}:\left(c_{00},\|\cdot\|_{y}\right) \rightarrow\left(\mathcal{B},|\cdot|_{y}\right)$ is an isometric embedding. For this purpose we will need to prove (3), (4) and (5) which follow.

(3) For every $x=(x(i)), z=(z(i)) \in c_{00}$ we have that $\sum_{i=1}^{\infty} x(i) z(i)=\int_{0}^{\infty} \phi(x) \phi(z)$. 
Indeed,

$$
\begin{aligned}
\sum_{i=1}^{\infty} x(i) z(i) & =\sum_{i=1}^{\infty} \int_{[i-1, i)} \sum_{j=1}^{\infty} x(j) \chi_{[j-1, j)} \sum_{k=1}^{\infty} z(k) \chi_{[k-1, k)} \\
& =\sum_{i=1}^{\infty} \int_{[i-1, i)} \phi(x) \phi(z) \\
& =\int_{0}^{\infty} \phi(x) \phi(z) .
\end{aligned}
$$

For all $n \in \mathbb{N} \cup\{0\}$ we have that $\phi\left(K_{n}\right) \subset L_{n}$.

In order to prove (4) we proceed by induction on $n$. For $n=0$ and $z \in K_{n}$ we have that $z=\lambda e_{k}$ where $|\lambda|=1$ and $k \in \mathbb{N}$. Thus $\phi(z)=\lambda \chi_{[k-1, k)} \in L_{0}$. Assume that (4) is valid for some $n \in \mathbb{N} \cup\{0\}$ and let $z \in K_{n+1}$. There exist $z_{1}=\left(z_{1}(i)\right)<z_{2}=\left(z_{2}(i)\right)<\cdots<z_{N}=$ $\left(z_{N}(i)\right)$ in $K_{n}$ such that $z=\sum_{i=1}^{N} y(i) z_{i}=\sum_{i=1}^{N} y(i) \sum_{j=1}^{\infty} z_{i}(j) e_{j}$ (where $\left(e_{j}\right)$ denotes the standard unit vector basis of $\left.\ell_{2}\right)$. Then $\phi(z)=\sum_{i=1}^{N} y(i) \sum_{j=1}^{\infty} z_{i}(j) \chi_{[j-1, j)}$. Notice that for all $i \in\{1, \ldots, N\}, \sum_{j=1}^{\infty} z_{i}(j) \chi_{[j-1, j)}=\phi\left(z_{i}\right) \in K_{n}$ and

$\sum_{j=1}^{\infty} z_{1}(j) \chi_{[j-1, j)}<\max \left(\operatorname{supp}\left(z_{1}\right)\right) \leq \sum_{j=1}^{\infty} z_{2}(j) \chi_{[j-1, j)}<\max \left(\operatorname{supp}\left(z_{2}\right)\right) \leq \cdots \leq \sum_{j=1}^{\infty} z_{N}(j) \chi_{[j-1, j)}$.

Thus $\phi(z) \in L_{n+1}$ which finishes the inductive proof of (4).

For every $n, m, \ell \in \mathbb{N} \cup\{0\}, x \in c_{00}$, with $m<\ell$ we have that

$$
\sup _{z \in K_{n}} \int_{m}^{\ell} \phi(x) \phi(z)=\sup _{g \in L_{n}} \int_{m}^{\ell} \phi(x) g \text {. }
$$

Notice that by (4) we obtain " $\leq$ " in (5). In order to prove " $\geq$ " in (5) we use induction on $n$. Let $n=0, g \in L_{n}$ and $m<\ell$ in $\mathbb{N}$. There exists $k \in \mathbb{N} \operatorname{such}$ that $\operatorname{supp}(g) \subseteq[k-1, k)$ and $\|g\|_{2} \leq 1$. Thus $\int_{m}^{\ell} \phi(x) g=x(k) \int_{[m, \ell) \cap[k-1, k)} g$ since $\phi(x)$ is constant on $[k-1, k)$. The last expression is equal to zero if $[m, \ell) \cap[k-1, k)=\emptyset$. If $[m, \ell) \cap[k-1, k) \neq \emptyset$ then $[k-1, k) \subseteq[m, \ell)$ and the last expression is dominated by

$$
|x(k)| \int_{[k-1, k)}|g| \leq|x(k)|\left(\int_{[k-1, k)}|g|^{2}\right)^{1 / 2} \leq|x(k)|=\int_{[k-1, k)} x(k) \phi(z)=\int_{m}^{\ell} \phi(x) \phi(z)
$$

where $z=(z(i)) \in K_{0}$ is defined by $z(i)=0$ for all $i \neq k, z(k)=|x(k)| / x(k)$ if $x \neq 0$, $z(k)=0$ if $x=0$. Assume that (5) is valid for some $n \in \mathbb{N} \cup\{0\}$. Let $g \in L_{n+1}$ and $m<\ell$ in $\mathbb{N}$. There exist $g_{1}, g_{2}, \ldots, g_{N} \in L_{n}$ and $\left(n_{i}\right)_{i=1}^{N-1} \subset \mathbb{N}$ such that $\operatorname{supp}\left(g_{1}\right)<n_{1} \leq \operatorname{supp}\left(g_{2}\right)<$ $n_{2} \leq \cdots<n_{N-1} \leq \operatorname{supp}\left(g_{N}\right)$ and $g=\sum_{i=1}^{N} y(i) g_{i}$. Thus if we set $n_{0}=0$ and we adopt the 
convention that $[s, t)=\emptyset$ whenever $s \geq t$, then

$$
\begin{aligned}
\int_{[m, \ell)} \phi(x) g & =\sum_{i=1}^{N} y(i) \int_{[m, \ell)} \phi(x) g_{i} \\
& =\sum_{i=1}^{N} y(i) \int_{\left[m \vee n_{i-1}, \ell \wedge n_{i}\right)} \phi(x) g_{i} \\
& \leq \sum_{i=1}^{N} y(i) \sup _{z_{i} \in K_{n}} \int_{\left[m \vee n_{i-1}, \ell \wedge n_{i}\right)} \phi(x) \phi\left(z_{i}\right),
\end{aligned}
$$

where the last inequality follows by the inductive hypothesis. Thus we continue our estimates as follows:

$$
\begin{aligned}
\int_{[m, \ell)} \phi(x) g & =\sum_{i=1}^{N} y(i) \underset{\substack{z_{i} \in K_{n} \\
\operatorname{supp}\left(z_{i}\right) \in\left[n_{i-1}+1, n_{i}\right]}}{\sup _{[m, \ell)}} \phi(x) \phi\left(z_{i}\right) \\
& \leq \sup \left\{\int_{[m, \ell)} \phi(x) \phi\left(\sum_{i=1}^{N} y(i) z_{i}\right): z_{i} \in K_{n} \text { for all } i,\right. \text { and } \\
& \left.\operatorname{supp}\left(z_{1}\right) \leq \tilde{n}_{1}<\operatorname{supp}\left(z_{2}\right) \leq \tilde{n}_{2}<\cdots \leq \operatorname{supp}\left(z_{N}\right) \text { for some }\left(\tilde{n}_{i}\right) \subseteq \mathbb{N}\right\} \\
& =\sup _{z \in K_{n+1}} \int_{[m, \ell)} \phi(x) \phi(z)
\end{aligned}
$$

which finishes the inductive proof of $(5)$.

Now, by combining (3) and (5) we obtain that $\phi \uparrow_{c_{00}}:\left(c_{00},\|\cdot\|_{y}\right) \rightarrow\left(\mathcal{B},|\cdot|_{y}\right)$ is an isometric embedding. Indeed, for every $x=(x(i)) \in c_{00}$ we have that

$$
\begin{aligned}
\|x\|_{y} & =\sup _{z=(z(i)) \in K} \sum_{i=1}^{\infty} x(i) z(i) \\
& =\sup _{z=(z(i)) \in K} \int_{0}^{\infty} \phi(x) \phi(z)(\text { by }(3)) \\
& =\sup _{g \in L} \int_{0}^{\infty} \phi(x) g(\text { by }(5)) \\
& =|\phi(x)|_{y} .
\end{aligned}
$$

Step 2: We define a norm $\|\cdot\| \|_{y}$ on $L_{2}[0, \infty)$ in terms of its dual ball as follows. Let

$$
L_{0}^{\prime}=\left\{f \in L_{2}[0, \infty):\|f\|_{2} \leq 1 \text { and } \operatorname{supp}(f) \subseteq[\alpha, \alpha+1) \text { for some } \alpha \geq 0\right\} .
$$

If $n \in \mathbb{N} \cup\{0\}$ and $L_{n}^{\prime}$ has been defined then

$$
L_{n+1}^{\prime}=L_{n}^{\prime} \cup\left\{\sum_{i=1}^{\infty} y(i) f_{i}: f_{i} \in L_{n}^{\prime} \text { for all } i \text { and } \operatorname{supp}\left(f_{1}\right)<\operatorname{supp}\left(f_{2}\right)<\cdots<\operatorname{supp}\left(f_{N}\right)\right\} \text {. }
$$


Let $L^{\prime}=\cup_{n=0}^{\infty} L_{n}^{\prime}$ and for a function $f \in L_{2}[0, \infty)$ define

$$
\|f \mid\| \|_{y}=\sup _{g \in L^{\prime}} \int_{0}^{\infty} f g .
$$

Notice that $L^{\prime}$ is a subset of the unit ball of $\left(L_{2}[0, \infty),\|\cdot\|_{2}\right)$ thus \|\|$\cdot\|\|_{y} \leq\|\cdot\|_{2}$. Also notice that for every $g \in L^{\prime}$ there exists a finite tree $\mathcal{T}$ and functions $\left(g_{t}\right)_{t \in \mathcal{T}}$ with

$$
\begin{aligned}
& g_{t}=\sum_{i=1}^{N} y(i) g_{t_{i}} \text { with } \operatorname{supp}\left(g_{t_{1}}\right)<\operatorname{supp}\left(g_{t_{2}}\right)<\cdots<\operatorname{supp}\left(g_{t_{N}}\right) \\
& \text { for every } t \in \mathcal{T} \backslash \max (\mathcal{T}),
\end{aligned}
$$

(where $t^{+}=\left\{t_{1}, \ldots, t_{N}\right\}$ ), and

$$
\begin{aligned}
& \left\|g_{t}\right\|_{2} \leq 1 \text { and there exists } \alpha_{t} \geq 0 \text { such that } \operatorname{supp}\left(g_{t}\right) \subseteq\left[\alpha_{t}, \alpha_{t}+1\right) \\
& \text { for every } t \in \max (\mathcal{T}) \text {. }
\end{aligned}
$$

We now show that $L^{\prime}$ is a dense subset of $\left\{f \in \mathcal{B}:\|f\|_{2} \leq 1\right\}$. Since $\left\{f \in \mathcal{B}:\|f\|_{2} \leq\right.$ $1\}$ is a dense subset of the unit ball of $\left(L_{2}[0, \infty),\|\cdot\|_{2}\right)$, we obtain that \|\|$\cdot\|\|_{y}$ is equal to $\|\cdot\|_{2}$. Now let $f \in \mathcal{B}$ with $\|f\|_{2} \leq 1$. We define a (perhaps infinite) tree $\mathcal{T}$, a $\mathcal{T}$ decomposition $\left(E_{t}\right)_{t \in \mathcal{T}}$ of $[\inf (\operatorname{supp}(f)), \sup (\operatorname{supp}(f)))$, and for every $t \in \mathcal{T}$ we define $f_{t} \in$ $L_{2}[0, \infty)$ with $\operatorname{supp}\left(f_{t}\right) \subseteq E_{t}$ as follows. Let $f_{\emptyset}=f$ and $E_{\emptyset}=[\inf (\operatorname{supp}(f)), \sup (\operatorname{supp}(f)))$. Assume that $f_{t}$ has been defined for some $t \in \mathcal{T}$, and $\left\|f_{t}\right\|_{2}=\|f\|_{2}$. Then $t$ is a maximal node of $\mathcal{T}$ if there exists $\alpha \geq 0$ such that $\operatorname{supp}\left(f_{t}\right) \subseteq[\alpha, \alpha+1)$. Otherwise, let $\alpha_{0}=$ $\inf \left(E_{t}\right)$ and define $\alpha_{0}<\alpha_{1}<\alpha_{2}<\cdots<\alpha_{N}=\sup \left(E_{t}\right)$ (where $N=\# \operatorname{supp}(y)$ ) such that $\left\|f_{t} \chi_{\left[\alpha_{i-1}, \alpha_{i}\right)}\right\|_{2}=y(i)\left\|f_{t}\right\|_{2}$ for all $i=1, \ldots, N$. Notice that this task is feasible since $\sum_{i=1}^{n} y(i)^{2}=1$ and the function $\alpha \mapsto\left\|f_{t} \chi_{\left[\alpha_{i-1}, \alpha\right)}\right\|_{2}$ is continuous. Then define $E_{t_{i}}=\left[\alpha_{i-1}, \alpha_{i}\right)$ and $f_{t_{i}}=\left\|f_{t}\right\|_{2} f_{t} \chi_{\left[\alpha_{i-1}, \alpha_{i}\right)} /\left\|f_{t} \chi_{\left[\alpha_{i-1}, \alpha_{i}\right)}\right\|_{2}$ for $i=1, \ldots, N$ and $t^{+}=\left\{t_{1}, \ldots, t_{N}\right\}$. Notice that $\left\|f_{t_{i}}\right\|_{2}=\left\|f_{t}\right\|_{2}=\|f\|_{2}$ for all $i$, and

$$
\sum_{i=1}^{N} y(i) f_{t_{i}}=\sum_{i=1}^{N} y(i)\left\|f_{t}\right\|_{2} \frac{f_{t} \chi_{\left[\alpha_{i-1}, \alpha_{i}\right)}}{\left\|f_{t} \chi_{\left[\alpha_{i-1}, \alpha_{i}\right)}\right\|_{2}}=\sum_{i=1}^{N} f_{t} \chi_{\left[\alpha_{i-1}, \alpha_{i}\right)}=f_{t} .
$$

It is easy to see by induction on $n=0,1,2, \ldots$ that for every $t \in \mathcal{T}$ which has $n$ predecessors, $\left\|f \chi_{E_{t}}\right\|_{2}=\|f\|_{2} y\left(j_{1}\right) y\left(j_{2}\right) \cdots y\left(j_{n}\right)$ for some $j_{1}, j_{2}, \ldots, j_{n} \in\{1, \ldots, N\}$ (which depend on $t$ ). Thus

$$
\left\|f \chi_{E_{t}}\right\| \rightarrow 0 \text { as } n \text { (the number of predecessors of } t \text { ) tends to infinity. }
$$

Notice also that for every two incomparable nodes $t, s \in \mathcal{T}$ we have that the intervals $E_{t}$ and $E_{s}$ are disjoint. Thus there exist at most $\sup \left(E_{\emptyset}\right)-\inf \left(E_{\emptyset}\right)$ many $t$ 's in $\mathcal{T}$ such that the length of $E_{t}$ is larger than 1 . Thus by (9) we obtain that

$$
g:=f-\sum_{\left(\text {length of } E_{t}\right)>1} f \chi_{E_{t}}+\sum_{\text {(length of } \left.E_{t}\right)>1}\left\|f \chi_{E_{t}}\right\|_{2}^{2} \chi_{\left[\inf \left(E_{t}\right), \inf \left(E_{t}\right)+1\right)}
$$

is a good approximant of $f$ if every $t \in \mathcal{T}$ for which (length of $E_{t}$ ) $>1$ has sufficiently large number of predecessors. Moreover, working as above we obtain a finite tree $\mathcal{T}$ and functions $\left(g_{t}\right)_{t \in \mathcal{T}}$ which satisfy (6), (7) and (8). Thus $g \in L^{\prime}$ which implies that $L^{\prime}$ is dense in $\left\{f \in \mathcal{B}:\|f\|_{2} \leq 1\right\}$. 
Step 3: We now prove that $\left.|||\cdot|\right|_{y}$ is equivalent to $|\cdot|_{y}$. Obviously we have that $|\cdot|_{y} \leq\|\| \cdot \mid \|_{y}$. Let $a:=\min _{1 \leq i \leq N} y(i)$ and $b:=\max _{1 \leq i \leq N} y(i)$. Let $M \in \mathbb{N}$ such that $b^{M} \leq a$. We will show that every function $g \in L^{\prime}$, the norming set of $\|\cdot\| \|_{y}$, can be written as a sum of at most $(M+4) N$ many functions from $L$ which will finish the proof. Fix $g \in L^{\prime}$ and consider an $N$-tree $\mathcal{T}$ and functions $\left(g_{t}\right)_{t \in \mathcal{T}} \subset L_{2}[0, \infty)$ which satisfy (6), (7) and (8). In particular, $\left(g_{t}\right)_{t \in \mathcal{T}}$ is a $\mathcal{T}$-decomposition of $g$. By (8), for every $t \in \max (\mathcal{T})$ there exists $\alpha_{t} \geq 0$ such that $\operatorname{supp}\left(g_{t}\right) \subseteq\left[\alpha_{t}, \alpha_{t}+1\right)$. Notice that if $\alpha_{t} \in \mathbb{N} \cup\{0\}$ for every $t \in \max (\mathcal{T})$ and moreover for every $s, t \in \max (\mathcal{T})$ with $s \neq t$ we have that $\alpha_{t} \neq \alpha_{s}$ then $g \in L$. For every $i=1,2, \ldots, N$ let $\mathcal{T}_{i} \subseteq \mathcal{T}$ to be the smallest tree which contains all maximal nodes of $\mathcal{T}$ of the form $\left(n_{1}, \ldots, n_{k}, i\right)$. Thus all maximal nodes of $\mathcal{T}_{i}$ have the form $\left(n_{1}, \ldots, n_{k}, i\right)$ for some $k \in \mathbb{N}$ and $n_{j} \in\{1, \ldots, N\}$ for $1 \leq j \leq k$. For every $t \in \mathcal{T}$ let $E_{t}$ to be the smallest interval (closed from the left, open from the right) containing the support of $g_{t}$. For $t \in \mathcal{T} \backslash \max (\mathcal{T})$ we have that the length of $E_{t}$ is larger than 1 . For every $i=1, \ldots, N$ let $A_{i}, B_{i} \subseteq \max \left(\mathcal{T}_{i}\right)$ with $A_{i} \cup B_{i}=\max \left(\mathcal{T}_{i}\right)$ be defined by $A_{i}=\left\{t \in \max \left(\mathcal{T}_{i}\right): E_{t} \cap \mathbb{N}=\emptyset\right\}$ and $B_{i}=\max \left(\mathcal{T}_{i}\right) \backslash A_{i}$. For every $t \in B_{i}$ let $n(t) \in \mathbb{N} \cap E_{t}$, (such $n(t)$ is unique since the length of $E_{t}$ is at most equal to 1$)$. We have that

$\operatorname{supp}(g) \subseteq \bigcup_{i=1}^{N}\left\{\bigcup\left\{E_{t}: t \in A_{i}\right\} \cup \bigcup\left\{E_{t} \cap[0, n(t)): t \in B_{i}\right\} \cup \bigcup\left\{E_{t} \cap[n(t), \infty): t \in B_{i}\right\}\right\}$.

For $i \in\{1, \ldots, N\}$ let $F_{i}$ be the restriction of $g$ on $\cup\left\{E_{t}: t \in A_{i}\right\}, G_{i}$ be the restriction of $g$ on $\cup\left\{E_{t} \cap[0, n(t)): t \in B_{i}\right\}$ and $H_{i}$ be the restriction of $g$ on $\cup\left\{E_{t} \cap[n(t), \infty): t \in B_{i}\right\}$. Notice that for any fixed $i \in\{1, \ldots, N\}$, any two intervals of the form $E_{t} \cap[0, n(t))$, where $t \in B_{i}$, are separated with an integer. The same is true for any two intervals of the form $E_{t} \cap[n(t), \infty)$, where $t \in B_{i}$. Thus for $i \in\{1, \ldots, N\}$, each of the functions $G_{i}$ and $H_{i}$ has a $\mathcal{T}_{i}$-tree decomposition (naturally inherited from the $\mathcal{T}$-tree decomposition of $g$ ), such that if $\left(G_{i, t}\right)_{t \in \mathcal{T}_{i}}$ and $\left(H_{i, t}\right)_{t \in \mathcal{T}_{i}}$ are the tree decompositions of $G_{i}$ and $H_{i}$ respectively, then the following is satisfied. For any $s, t \in \max \left(\mathcal{I}_{i}\right)$ with $s \neq t$ and $G_{i, t}, G_{i, s} \neq 0$, we have that the supports of $G_{i, s}$ and $G_{i, t}$ are separated by an integer. Similarly, for any $s, t \in \max \left(\mathcal{T}_{i}\right)$ with $s \neq t$ and $H_{i, t}, H_{i, s} \neq 0$, we have that the supports of $H_{i, s}$ and $H_{i, t}$ are separated by an integer. Thus for $i \in\{1, \ldots, N\}, G_{i}, H_{i}$ belong to $L$, the norming set for $|\cdot|_{y}$.

Unfortunately we cannot say the same about $F_{i}$ ! Indeed for $i \in\{1, \ldots N\}$, let $\left(F_{i, t}\right)_{t \in \max } \mathcal{T}_{i}$ be the $\mathcal{T}_{i}$-tree decomposition of $F_{i}$ which is naturally inherited by the $\mathcal{T}$-tree decomposition of $g$. In general for $s, t \in \max \left(\mathcal{T}_{i}\right)$ with $s \neq t$, the supports of $F_{i, s}$ and $F_{i, t}$ may not be separated by an integer. Indeed, let $s, t \in \max \left(\mathcal{T}_{i}\right)$ be such that $s \neq t, F_{i, t} \neq 0, F_{i, s} \neq 0$ and the sets $\left(s^{-}\right)^{+}$and $\left(t^{-}\right)^{+}$contain only maximal elements of $\mathcal{T}_{i}$. These conditions guarantee that $E_{t^{-}}$and $E_{s^{-}}$are disjoint intervals, each of length larger than 1 . Also $\emptyset \neq E_{t} \subseteq E_{t^{-}}$ and $\emptyset \neq E_{s} \subseteq E_{s^{-}}$. It is easy to see that these conditions do not imply that $E_{t}$ and $E_{s}$ are separated by an integer. On the other hand there are no three different nodes $s, t, w \in \max \left(\mathcal{T}_{i}\right)$ so that $F_{i, s}, F_{i, t}, F_{i, w} \neq 0$, the sets $\left(s^{-}\right)^{+},\left(t^{-}\right)^{+},\left(w^{-}\right)^{+}$contain only maximal nodes of $\mathcal{T}_{i}$ and $E_{t}, E_{s}, E_{w}$ are contained in the same integer interval (i.e. an interval of the form $[m, m+1)$ for some $m \in \mathbb{N} \cup\{0\})$. Thus if from each integer interval we extract the "most left" and the "most right" interval $E_{t}$ for $t \in \max \left(\mathcal{T}_{i}\right)$, then there is no other node $s$ in $\max \left(\mathcal{T}_{i}\right)$ such that $E_{s} \neq \emptyset,\left(s^{-}\right)^{+}$has only maximal nodes and $E_{s} \subseteq[m, m+1)$. This is the task of the next two paragraphs. For the rest of the proof, fix $i \in\{1, \ldots, N\}$ and let $\left(F_{i, t}\right)_{t \in \mathcal{T}_{i}}$ be the natural $\mathcal{T}_{i}$-tree decomposition of $F_{i}$ which is inherited by the $\mathcal{T}$-decomposition of $g$. 
Fix $t \in A_{i}$. There exists a unique integer interval $[m, m+1)$ with $E_{t} \subseteq[m, m+1)$. The node $t$ will be called left (perhaps the term $i$-left would be more precise, but since $i$ is fixed we do not want to make the terminology too long) if there is no $s \in A_{i}$ such that $m \leq E_{s}<E_{t}$ and $E_{s}$ is non-empty. Let $L_{i}$ be the restriction of $F_{i}$ on $\cup\left\{E_{t}: t\right.$ is left $\}$. The function $L_{i}$ has a $\mathcal{T}_{i}$-tree decomposition $\left(L_{i, t}\right)_{t \in \mathcal{T}_{i}}$ which is naturally inherited by the $\mathcal{T}_{i}$-tree decomposition of $F_{i}$. For any two left nodes $t_{1}, t_{2}$ with $L_{i, t_{1}}, L_{i, t_{2}} \neq 0$, we have that the supports of $L_{i, t_{1}}$ and $L_{i, t_{2}}$ are separated by an integer, thus $L_{i}$ belongs to $L$, the norming set for $|\cdot|_{y}$.

Fix $t \in A_{i}$ and let $[m, m+1)$ be the unique integer interval with $E_{t} \subseteq[m, m+1)$. The node $t$ will be called right if $t$ is not left and there is no $s \in A_{i}$ such that $E_{t}<E_{s}<m+1$ and $E_{s}$ is non-empty. Let $R_{i}$ be the restriction of $F_{i}$ on $\cup\left\{E_{t}: t\right.$ is right $\}$. Arguing similarly as we $\operatorname{did}$ for $L_{i}$, we have that $R_{i} \in L$.

Let $\tilde{F}_{i}:=F_{i}-\left(L_{i}+R_{i}\right)$ and $\left(\tilde{F}_{i, t}\right)_{t \in \mathcal{T}_{i}}$ be the $\mathcal{T}_{i}$-tree decomposition of $\tilde{F}_{i}$ which is naturally inherited by the $\mathcal{T}_{i}$-tree decomposition of $F_{i}$. For $s, t \in \max \left(\mathcal{T}_{i}\right)$ with $s \neq t$ and $\tilde{F}_{i, s}, \tilde{F}_{i, t} \neq 0$, the supports of $F_{i, s}$ and $F_{i, t}$ may not be separated by an integer! For instance consider the following scenario. Consider two maximal nodes $s, t$ of $\mathcal{T}_{i}$ with $s^{-} \prec t^{-}$. Since $\operatorname{supp}\left(\tilde{F}_{i, t^{-}}\right) \subset \operatorname{supp}\left(\tilde{F}_{i, s^{-}}\right)$, and $\operatorname{supp}\left(\tilde{F}_{i, t^{-}}\right)$is not contained in any integer interval we have that $\operatorname{supp}\left(\tilde{F}_{i, s^{-}}\right)$is not contained in any integer interval either. Obviously the inclusions $\operatorname{supp}\left(\tilde{F}_{i, s}\right) \subseteq \operatorname{supp}\left(\tilde{F}_{i, s^{-}}\right)$and $\operatorname{supp}\left(\tilde{F}_{i, t}\right) \subseteq \operatorname{supp}\left(\tilde{F}_{i, t^{-}}\right)$do not imply that the sets $\operatorname{supp}\left(\tilde{F}_{i, s}\right)$ and $\operatorname{supp}\left(\tilde{F}_{i, t}\right)$ are separated by an integer! The special nodes $s, t$ that we just considered have to be treated in a special way, and that is what we do in what follows.

A sequence $\left(t_{1}, t_{2}, \ldots, t_{k}\right) \subset \mathcal{T}_{i},(k \geq 2)$, is called $i$-special if the following are satisfied:

(a) $t_{j} \in \max \left(\mathcal{T}_{i}\right)$ for $1 \leq j \leq k$.

(b) $t_{1}^{-} \prec t_{2}^{-} \prec \cdots \prec t_{k}^{-}$.

(c) The sets $\operatorname{supp}\left(\tilde{F}_{i, t_{j}}\right)$ for $j \in\{1, \ldots, k\}$ are all contained in the same integer interval.

(d) None of the $t_{j}$ 's is left or right.

(e) The sequence $\left(t_{1}, t_{2}, \ldots, t_{k}\right)$ is maximal with the properties (a), (b), (c) and (d) (i.e. it is not properly contained in any sequence which satisfies (a), (b), (c) and (d)).

Properties (c), (d) and (e) ensure that any two $i$-special sequences are disjoint.

Define $\mathcal{T}_{i, 1}$ to be the smallest subtree of $\mathcal{T}_{i}$ satisfying the following two conditions:

(i) If $t \in \max \left(\mathcal{T}_{i}\right)$ and $t$ does not belong in any $i$-special sequence then $t \in \mathcal{T}_{i, 1}$.

(ii) For any $i$ special sequence $\left(t_{1}, \ldots, t_{k}\right)$ we have that $t_{1} \in \mathcal{T}_{i, 1}$.

Recall that in the beginning of Step 3 we defined $a, b$ and $M$ by: $a:=\min _{1 \leq \ell \leq N} y(\ell)$, $b:=\max _{1 \leq \ell \leq N} y(\ell)$ and $M \in \mathbb{N}$ such that $b^{M} \leq a$. For $j \in\{2, \ldots, M-1\}$ let $\overline{\mathcal{T}}_{i, j}$ be the smallest subtree of $\mathcal{T}_{i}$ whose maximal nodes are the elements $t_{j}$ of any $i$-special sequence $\left(t_{1}, \ldots, t_{k}\right)$ with $k \geq j$. By the definition of $\mathcal{T}_{i, j}$ for $1 \leq j \leq M-1$ we have that if $\left(t_{1}, \ldots, t_{k}\right)$ is an $i$-special sequence and $1 \leq j \leq k$ then $t_{\ell} \notin \mathcal{T}_{i, j}$ for $\ell \in\{1, \ldots k\} \backslash\{j\}$. For every $j \in\{1, \ldots, M-1\}$ let $\tilde{F}_{i, j}$ be the restriction of $\tilde{F}_{i}$ on $\cup\left\{E_{t}: t \in A_{i} \cap \mathcal{T}_{i, j}\right\}$. Hence $\tilde{F}_{i, j}$ has a $\mathcal{T}_{i, j}$-tree decomposition $\left(\tilde{F}_{i, j, t}\right)_{t \in \mathcal{T}_{i, j}}$ which is naturally inherited by the $\mathcal{T}_{i}$-tree decomposition of $\tilde{F}_{i}$. Moreover, since $i$-special sequences are maximal, (see (e) in the definition of $i$-special sequences), we have that no two maximal nodes of $\mathcal{T}_{i, j}$ belong in the same $i$-special sequence. Hence for every two maximal nodes $s, t$ of $\mathcal{T}_{i, j}$ we have that the supports of $\tilde{F}_{i, j, s}$ and $\tilde{F}_{i, j, t}$ are separated by an integer. Thus $\tilde{F}_{i, j}$ belongs to the norming set $L$ of $|\cdot|_{y}$ for every $j \in\{1, \ldots, M-1\}$. 
Notice that $\cup_{j=1}^{M-1} \operatorname{supp}\left(\tilde{F}_{i, j}\right)$ may be a strict subset of $\operatorname{supp}\left(\tilde{F}_{i}\right)$ and thus $\tilde{F}_{i}$ may not be equal to $\sum_{j=1}^{M-1} \tilde{F}_{i, j}$. This will be the case if there are $i$-special sequences that have length $k \geq$ $M$. Now we take care of such long $i$-special sequences. Fix an $i$-special sequence $\left(t_{1}, \ldots, t_{k}\right)$ with $k \geq M$. Let $[m, m+1)$ be the unique integer interval with $\operatorname{supp}\left(\tilde{F}_{i, t_{j}}\right) \subseteq[m, m+1)$ for all $j \in\{1, \ldots, k\}$. Let $\tilde{F}_{i, t_{1}^{-}} \Upsilon_{[m, m+1)}$ and $\tilde{F}_{i, t_{M}^{-}} \Upsilon_{[m, m+1)}$ be the restrictions of $\tilde{F}_{i, t_{1}^{-}}$and $\tilde{F}_{i, t_{M}^{-}}$ respectively on $[m, m+1)$. Thus we can write

$$
\tilde{F}_{i, t_{1}^{-}} \Upsilon_{[m, m+1)}=\left(\prod y\left(n_{\alpha}\right)^{m_{\alpha}}\right) \tilde{F}_{i, t_{M}^{-}} \Upsilon_{[m, m+1)}
$$

where $n_{\alpha}, m_{\alpha} \in\{1, \ldots, N\}$ and the product $\prod y\left(n_{\alpha}\right)^{m_{\alpha}}$ has at least $M$-terms counting multiplicities. Thus $\prod y\left(n_{\alpha}\right)^{m_{\alpha}} \leq b^{M} \leq a \leq y(i)$. Hence $\left\|\tilde{F}_{i, t_{1}^{-}} \uparrow_{[m, m+1)}\right\|_{2} \leq y(i)\left\|\tilde{F}_{i, t_{M}^{-}} \Upsilon_{[m, m+1)}\right\|_{2}$ and $\left\|\tilde{F}_{i, t_{M}^{-}} \uparrow_{[m, m+1)}\right\|_{2} \leq\left\|\tilde{F}_{i, t_{M}^{-}}\right\|_{2} \leq 1$ by (8). Let $\mathcal{T}_{i, M}$ be the subtree of $\mathcal{T}_{i, 1}$ whose maximal nodes are the nodes $t_{1}$ for any $i$-maximal sequence $\left(t_{1}, \ldots, t_{k}\right)$ with $k \geq M$. Let

$$
\begin{gathered}
S_{i}:=\cup\left\{E_{t}: t \in A_{i} \text { and there exists an } i \text {-special sequence }\left(t_{1}, \ldots, t_{k}\right) \text { with } k \geq N\right. \\
\text { and } \left.M \leq j \leq k \text { such that } t=t_{j}\right\} .
\end{gathered}
$$

Thus the restriction $\tilde{F}_{i, M}:=\tilde{F}_{i}\left\lceil_{S_{i}}\right.$ of the function $\tilde{F}_{i}$ on $S_{i}$ has a $\mathcal{T}_{i, M}$-tree decomposition $\left(\tilde{F}_{i, M, t}\right)_{t \in \mathcal{T}_{i, M}}$, where for any $i$-special sequence $\left(t_{1}, \ldots, t_{k}\right)$ with $k \geq M$ we set $\tilde{F}_{i, M, t_{1}}:=$ $\tilde{F}_{i, t_{M}^{-}} \Upsilon_{[m, m+1)}$, (where $[m, m+1)$ is the unique integer interval which contains the sets $\operatorname{supp}\left(\tilde{F}_{i, t_{j}}\right)$ for $\left.j \in\{1, \ldots, k\}\right)$ and $\tilde{F}_{i, M, t_{1}^{-}}:=\tilde{F}_{i, t_{1}^{-}} \uparrow_{[m, m+1)}$. Hence $\tilde{F}_{i, M}$ belongs to $L$ the norming set of $|\cdot|_{y}$.

We have decomposed the arbitrary element $g$ of the norming set $L^{\prime}$ of \|\|$\cdot\|\|_{y}$ into the sum of the functions $G_{i}, H_{i}, L_{i}, R_{i}, \tilde{F}_{i, j}$ (where $i \in\{1, \ldots, N\}$ and $j \in\{1, \ldots, M\}$ ), a total of $(M+4) N$ many functions of the norming set $L$ of $|\cdot|_{y}$. This implies that $\left.\left|\||\cdot|\|_{y} \leq(M+4) N\right| \cdot\right|_{y}$ and finishes the proof.

\section{REFERENCES}

[1] Odell, E. ; Schlumprecht, Th. The distortion problem. Acta Math. 173 (1994), no. 2, 259-281.

[2] Odell, E. ; Schlumprecht, Th. Distortion and stabilized structure in Banach spaces; new geometric phenomena for Banach and Hilbert spaces. Proceedings of the International Congress of Mathematicians, Vol. 1, 2 (Zürich, 1994), 955-965, Birkhäuser, Basel, 1995.

[3] Bellenot, S.F. Tsirelson superspaces and $\ell_{p}$. J. Funct. Anal. 69 (1986), no. 2, 207-228.

[4] Bernués, J.; Deliyanni, I. Families of finite subsets of $\mathbb{N}$ of low complexity and Tsirelson type space, Math. Nachr. 222 (2001), 15-29.

[5] Gowers, W.T. Ramsey methods in Banach spaces. Handbook of the geometry of Banach spaces, Vol. 2, 1071-1097, North-Holland, Amsterdam, 2003.

[6] Pelczar, A.M. Stabilization of Tsirelson-type norms on $\ell_{p}$ spaces, preprint, avalaible at http://www.arxiv.org/pdf/math.FA/0508352

Department of Mathematics, University of South Carolina, Columbia, SC 29208.

E-mail address: giorgis@math.sc.edu, sanacory@math.sc.edu 